Acta Crystallographica Section E

Structure Reports

Online

ISSN 1600-5368

\section{2-(4-Bromobenzenesulfonamido)acetic acid}

\section{Muhammad Nadeem Arshad, ${ }^{a *}$ Islam Ullah Khan, ${ }^{a}$ Muhammad Shafiq, ${ }^{a}$ Muhammad Naeem Khan' ${ }^{b}$ and Helen Stoeckli-Evans ${ }^{\mathrm{c}}$}

aMaterials Chemistry Laboratory, Department of Chemistry, GC University, Lahore,

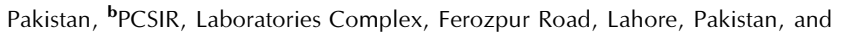
'Institute of Physics, Université of Neuchâtel, Rue Emile-Argand 11, CH-2009 Neuchâtel, Switzerland

Correspondence e-mail: mnachemist@hotmail.com

Received 30 June 2009; accepted 4 July 2009

Key indicators: single-crystal X-ray study; $T=296 \mathrm{~K}$; mean $\sigma(\mathrm{C}-\mathrm{C})=0.008 \AA$; $R$ factor $=0.058 ; w R$ factor $=0.168 ;$ data-to-parameter ratio $=18.7$.

The title compound, $\mathrm{C}_{8} \mathrm{H}_{8} \mathrm{BrNO}_{4} \mathrm{~S}$, a halogenated sulfonamide, was prepared by basic hydrolysis of the methyl ester. In the crystal, molecules form centrosymmetric hydrogenbonded dimers via the carboxyl groups. These dimers are further linked by $\mathrm{N}-\mathrm{H} \cdots \mathrm{O}$ interactions involving the carbonyl $\mathrm{O}$ and amide $\mathrm{H}$ atoms, forming a ribbon-like structure propagating in [010]. These ribbons are further linked via $\mathrm{C}-\mathrm{H} \cdots \mathrm{O}$ interactions, forming a three-dimensional network.

\section{Related literature}

For details of the crystal structure of the methyl ester of the title compound, see: Arshad et al. (2008b). For related structures, see: Arshad et al. (2008a); Arshad et al. (2009). For related thiazine heterocycles, see: Arshad et al. (2008c). For hydrogen-bonding patterns, see: Bernstein et al. (1995).<smiles>O=C(O)CNS(=O)(=O)c1ccc(Br)cc1</smiles>

\section{Experimental}

Crystal data

$\mathrm{C}_{8} \mathrm{H}_{8} \mathrm{BrNO}_{4} \mathrm{~S}$

$M_{r}=294.12$

Triclinic, $P \overline{1}$ $a=5.0042(4) \AA$

$b=7.9997(6) \AA$ $c=13.2289(11) \AA$

$$
\begin{aligned}
& \alpha=79.691(4)^{\circ} \\
& \beta=88.667(5)^{\circ} \\
& \gamma=81.404(4)^{\circ} \\
& V=515.18(7) \AA^{3} \\
& Z=2
\end{aligned}
$$

Data collection

Bruker Kappa APEXII CCD diffractometer

Absorption correction: multi-scan (SADABS; Bruker, 2007)

$T_{\min }=0.612, T_{\max }=0.632$

Refinement

$R\left[F^{2}>2 \sigma\left(F^{2}\right)\right]=0.058$

$w R\left(F^{2}\right)=0.168$

$S=0.95$

2557 reflections

Mo $K \alpha$ radiation

$\mu=4.18 \mathrm{~mm}^{-1}$

$T=296 \mathrm{~K}$

$0.28 \times 0.17 \times 0.11 \mathrm{~mm}$

10359 measured reflections 2557 independent reflections 1243 reflections with $I>2 \sigma(I)$ $R_{\text {int }}=0.056$

\section{Table 1}

Hydrogen-bond geometry $\left(\AA{ }^{\circ}\right)$.

\begin{tabular}{lllll}
\hline$D-\mathrm{H} \cdots A$ & $D-\mathrm{H}$ & $\mathrm{H} \cdots A$ & $D \cdots A$ & $D-\mathrm{H} \cdots A$ \\
\hline $\mathrm{O} 3-\mathrm{H} 3 O \cdots \mathrm{O} 4^{\text {i }}$ & 0.82 & 1.85 & $2.671(5)$ & 174 \\
$\mathrm{~N}^{1}-\mathrm{H} 1 \cdots \mathrm{O} 4^{\text {ii }}$ & 0.86 & 2.38 & $3.124(5)$ & 146 \\
$\mathrm{C} 2-\mathrm{H} 2 \cdots \mathrm{O} 1^{\text {iii }}$ & 0.93 & 2.53 & $3.384(7)$ & 153 \\
$\mathrm{C} 3-\mathrm{H} 3 \cdots 3^{\text {iv }}$ & 0.93 & 2.50 & $3.423(7)$ & 170
\end{tabular}

Symmetry codes: (i) $-x+1,-y-1,-z+1$; (ii) $-x+1,-y,-z+1$; (iii) $x, y+1, z$; (iv) $x-1, y+1, z$

Data collection: APEX2 (Bruker, 2007); cell refinement: SAINT (Bruker, 2007); data reduction: $S A I N T$; $\operatorname{program}(\mathrm{s})$ used to solve structure: SHELXS97 (Sheldrick, 2008); program(s) used to refine structure: SHELXL97 (Sheldrick, 2008); molecular graphics: PLATON (Spek, 2009) and Mercury (Macrae et al., 2006); software used to prepare material for publication: SHELXL97.

MNA acknowledges the Higher Education Commission of Pakistan for providing a PhD Scholarship under PIN 042120607-PS2-183.

Supplementary data and figures for this paper are available from the IUCr electronic archives (Reference: TK2493).

\section{References}

Arshad, M. N., Khan, I. U., Shafiq, M. \& Mukhtar, A. (2009). Acta Cryst. E65, 0549 .

Arshad, M. N., Khan, I. U. \& Zia-ur-Rehman, M. (2008a). Acta Cryst. E64, o2283-o2284.

Arshad, M. N., Tahir, M. N., Khan, I. U., Ahmad, E. \& Shafiq, M. (2008b). Acta Cryst. E64, o2380.

Arshad, M. N., Tahir, M. N., Khan, I. U., Shafiq, M. \& Siddiqui, W. A. (2008c). Acta Cryst. E64, o2045.

Bernstein, J., Davis, R. E., Shimoni, L. \& Chang, N.-L. (1995). Angew. Chem. Int. Ed. Engl. 34, 1555-1573.

Bruker (2007). APEX2, SADABS and SAINT. Bruker AXS Inc., Madison, Wisconsin, USA.

Macrae, C. F., Edgington, P. R., McCabe, P., Pidcock, E., Shields, G. P., Taylor, R., Towler, M. \& van de Streek, J. (2006). J. Appl. Cryst. 39, 453-457.

Sheldrick, G. M. (2008). Acta Cryst. A64, 112-122.

Spek, A. L. (2009). Acta Cryst. D65, 148-155. 


\section{supporting information}

Acta Cryst. (2009). E65, o1816 [doi:10.1107/S160053680902604X]

\section{2-(4-Bromobenzenesulfonamido)acetic acid}

\section{Muhammad Nadeem Arshad, Islam Ullah Khan, Muhammad Shafiq, Muhammad Naeem Khan and Helen Stoeckli-Evans}

\section{S1. Comment}

The title compound, (I), was prepared by basic hydrolysis of methyl (4-bromobenzenesulfonamido)acetate (II) (Arshad et al., 2008b), in a continuation of our studies on the synthesis of thiazine related heterocycles (Arshad et al., 2008c). We have previously reported the crystal structures of 2-(benzenesulfonamido)acetic acid (III) (Arshad et al., 2008a) and 2-(2iodobenzenesulfonamido)acetic acid (IV) (Arshad et al., 2009).

The molecular structure of (I), Fig. 1, reveals the bond lengths and angles are similar to those found for compounds (II), (III) and (IV).

The presence of the carboxylic acid group leads to the formation of characteristic $\mathrm{O}-\mathrm{H} \cdots \mathrm{O}$ hydrogen-bonded centrosymmetric dimers (Table 1 and Fig. 2). These dimers are linked via $\mathrm{N} 1-\mathrm{H} 1 \cdots \mathrm{O} 4$ interactions, involving the carbonyl $\mathrm{O}$-atom and the $\mathrm{H}$-atom of the amido group, to form a ribbon-like structure propagating in the [010] direction (Table 1 and Fig. 2). The ribbons are further linked by $\mathrm{C}-\mathrm{H} \cdots \mathrm{O}$ interactions to form a 3-D network (Table 1 and Fig. 3).

It is interesting to compare the hydrogen bonding patterns in the three acids; (I), (III) and (IV). The formation of the hydrogen bonded carboxylic acid dimers is the same in all three compounds, i.e. $R^{2}{ }_{2}(8)$ (Bernstein et al., 1995). The $\mathrm{N}-$ $\mathrm{H} \cdots \mathrm{O}$ hydrogen-bonding involves the sulfonamido groups in (III) and (IV) $\left[R^{2}{ }_{2}(8)\right]$, while in (I) it involves the carbonyl $\mathrm{O}$-atom $(\mathrm{O} 4)$ and the $\mathrm{H}$-atom of the amido group (Table 1). This leads to a larger hydrogen-bonded ring of the form $\left[R_{2}^{2}(10)\right]$, as shown in Fig. 4 .

\section{S2. Experimental}

Methyl (4-bromobenzenesulfonamido)acetate(II) (Arshad et al., 2008b) (1.0 g, $3.247 \mathrm{mmol}$ ) was dissolved in an aqueous sodium hydroxide solution $(10 \%, 10 \mathrm{ml})$. The resulting solution was refluxed for an hour. The reaction mixture was then cooled to room temperature and acidified with $1 \mathrm{~N} \mathrm{HCl}$. A white precipitate was obtained. This was filtered off, washed with distilled water and dried. Crystals were obtained by recrystallization from methanol.

\section{S3. Refinement}

The $\mathrm{H}$-atoms were included in calculated positions and treated as riding atoms: $\mathrm{O}-\mathrm{H}=0.82 \AA, \mathrm{N}-\mathrm{H}=0.86 \AA, \mathrm{C}-\mathrm{H}=$ $0.93-0.97 \AA$, with $U_{\text {iso }}(\mathrm{H})=\mathrm{k} \times U_{\text {eq }}($ parent O-, $\mathrm{N}-$ or C-atom), where $\mathrm{k}=1.5$ for $\mathrm{OH}$, and 1.2 for $\mathrm{N}$ - and C-bound $\mathrm{H}$ atoms.

The maximum and minimum residual electron density peaks of 1.15 and $-0.38 \mathrm{e} \AA^{-3}$, respectively, were located at $1.10 \AA$ and $0.78 \AA$, respectively, from atom $\mathrm{Br} 1$. 




\section{Figure 1}

The molecular structure of (I), with displacement ellipsoids drawn at the $50 \%$ probability level.

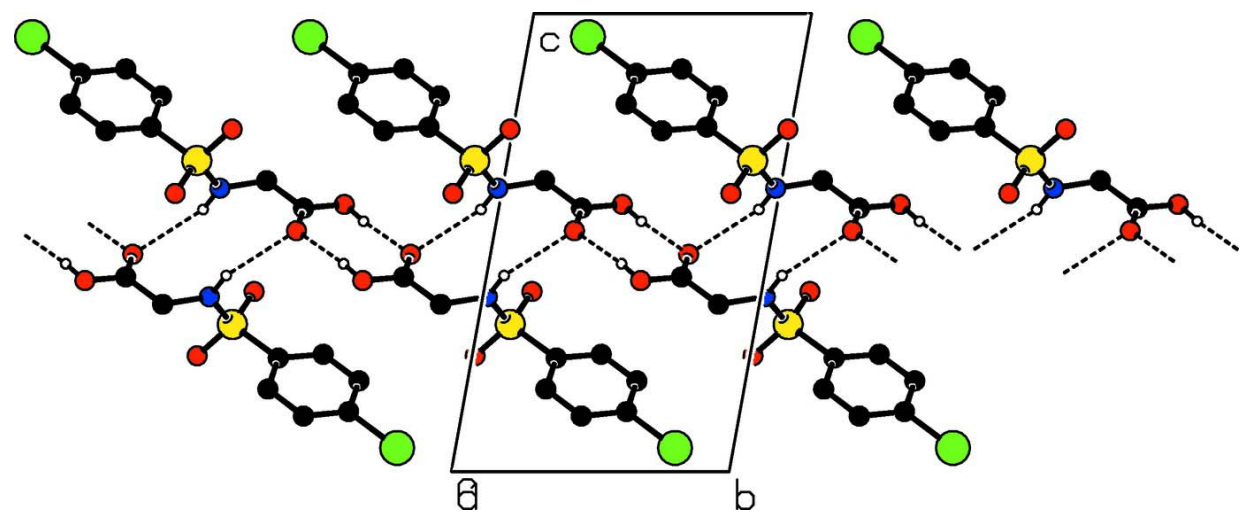

Figure 2

A view along the $a$ axis of the crystal packing of compound (I), with $\mathrm{O}-\mathrm{H} \cdots \mathrm{O}$ and $\mathrm{N}-\mathrm{H} \cdots \mathrm{O}$ hydrogen bonds drawn as dashed lines [see Table 1 for details; $\mathrm{H}$ atoms not involved in hydrogen bonding have been omitted for clarity]. 


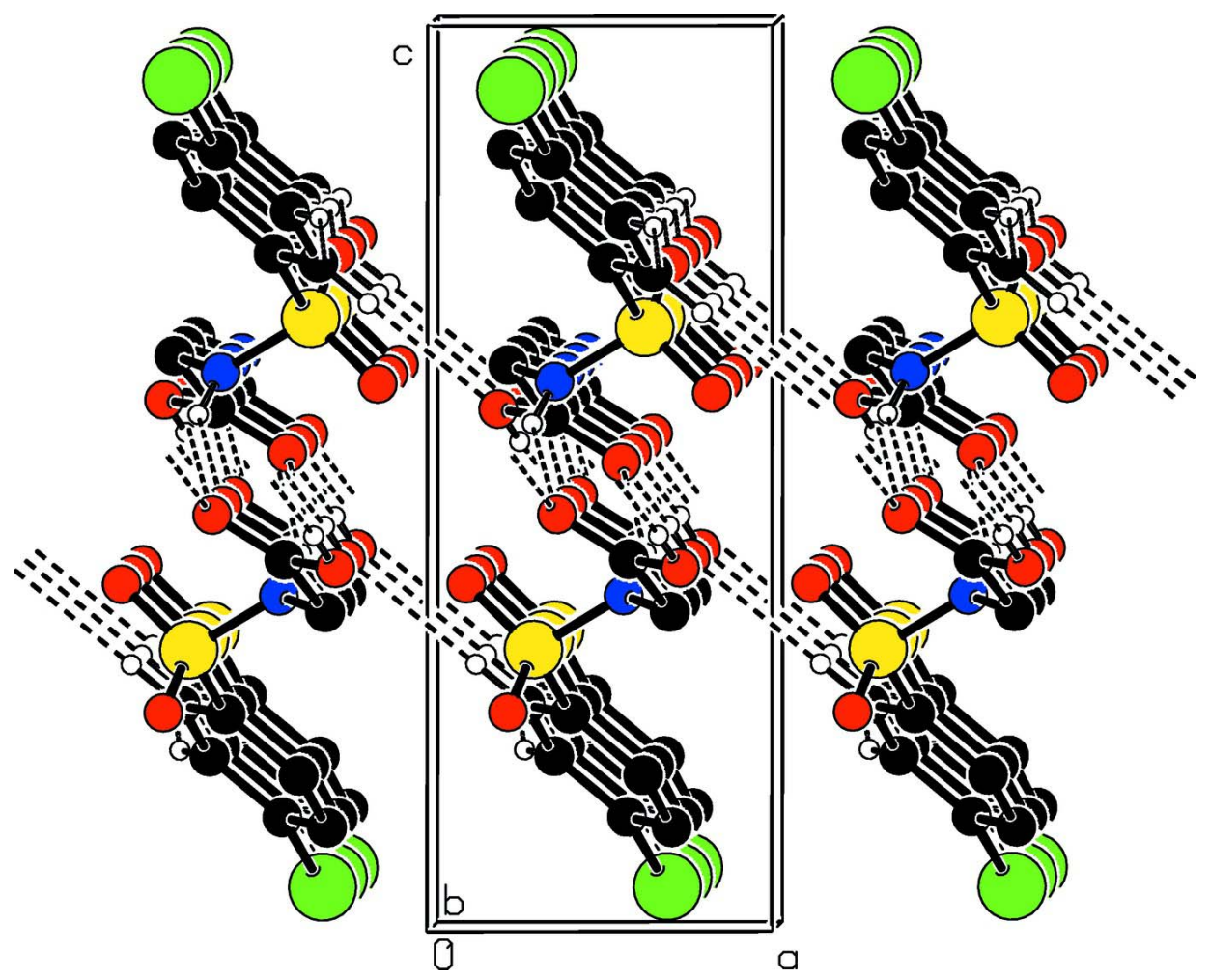

Figure 3

A view along the $b$ axis of the crystal packing of (I), with $\mathrm{O}-\mathrm{H} \cdots \mathrm{O}, \mathrm{N}-\mathrm{H} \cdots \mathrm{O}$ and $\mathrm{C}-\mathrm{H} \cdots \mathrm{O}$ hydrogen bonds drawn as dashed lines [see Table 1 for details; $\mathrm{H}$ atoms not involved in hydrogen bonding have been omitted for clarity]. 


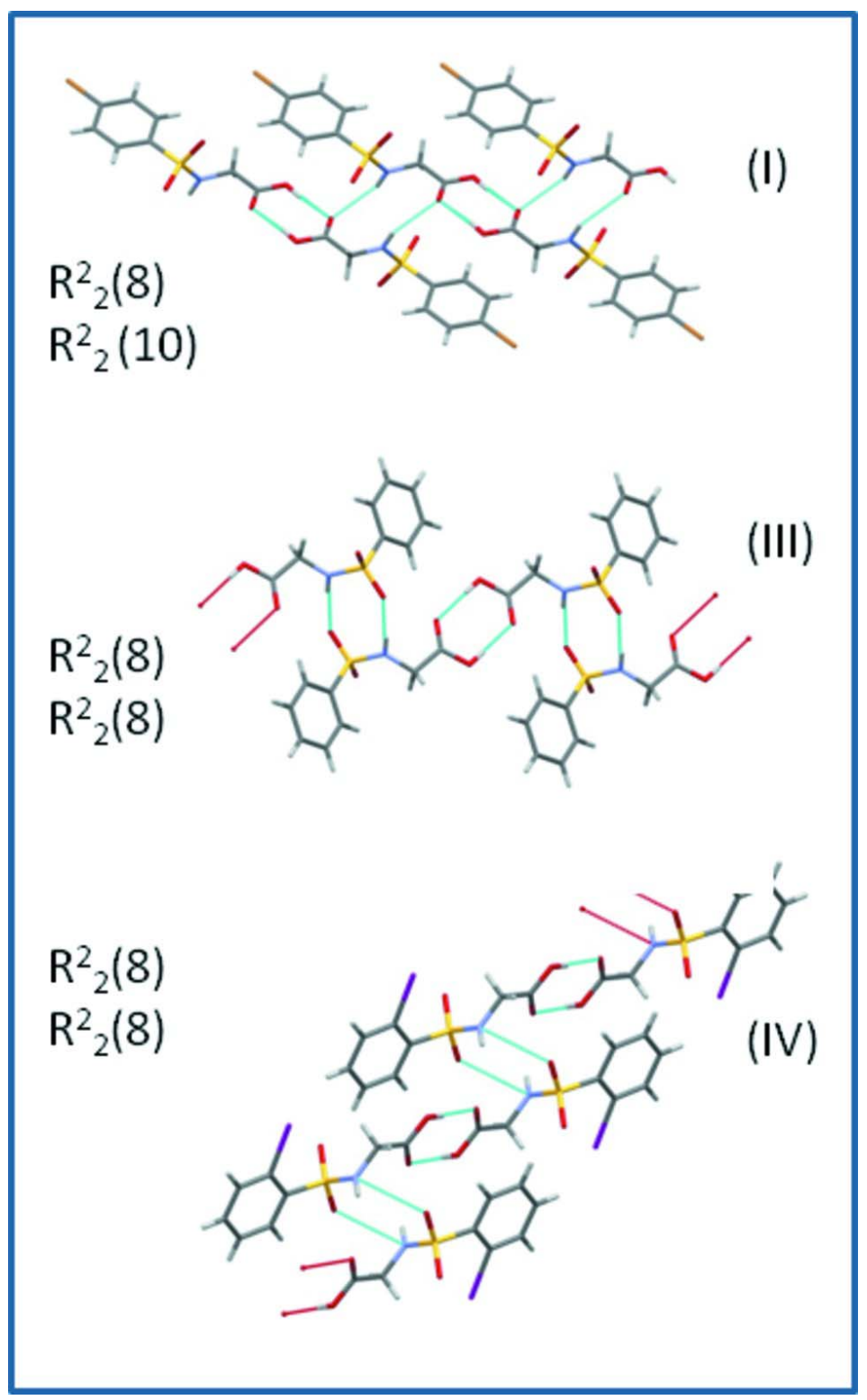

\section{Figure 4}

A view of the hydrogen bonding patterns in the three acid compounds: (I), (III) and (IV). The hydrogen bonds are shown as pale-blue lines.

\section{2-(4-Bromobenzenesulfonamido)acetic acid}

\section{Crystal data}

$\mathrm{C}_{8} \mathrm{H}_{8} \mathrm{BrNO}_{4} \mathrm{~S}$

$M_{r}=294.12$

Triclinic, $P \overline{1}$

Hall symbol: -P 1

$a=5.0042(4) \AA$

$b=7.9997(6) \AA$

$c=13.2289(11) \AA$

$\alpha=79.691(4)^{\circ}$

$\beta=88.667(5)^{\circ}$ $\gamma=81.404(4)^{\circ}$
$V=515.18(7) \AA^{3}$
$Z=2$
$F(000)=292$
$D_{\mathrm{x}}=1.896 \mathrm{Mg} \mathrm{m}^{-3}$

$D_{\mathrm{x}}=1.896 \mathrm{Mg} \mathrm{m}^{-3}$
Mo $K \alpha$ radiation, $\lambda=0.71073 \AA$

Cell parameters from 1869 reflections

$\theta=2.2-21.8^{\circ}$

$\mu=4.18 \mathrm{~mm}^{-1}$ 
$T=296 \mathrm{~K}$

Needle, colorless

Data collection

\section{Bruker Kappa APEXII CCD} diffractometer

Radiation source: fine-focus sealed tube Graphite monochromator $\varphi$ and $\omega$ scans

Absorption correction: multi-scan (SADABS; Bruker, 2007)

$T_{\min }=0.612, T_{\max }=0.632$

Refinement

Refinement on $F^{2}$

Least-squares matrix: full

$R\left[F^{2}>2 \sigma\left(F^{2}\right)\right]=0.058$

$w R\left(F^{2}\right)=0.168$

$S=0.95$

2557 reflections

137 parameters

0 restraints

Primary atom site location: structure-invariant direct methods
$0.28 \times 0.17 \times 0.11 \mathrm{~mm}$

10359 measured reflections

2557 independent reflections

1243 reflections with $I>2 \sigma(I)$

$R_{\text {int }}=0.056$

$\theta_{\max }=28.3^{\circ}, \theta_{\min }=2.6^{\circ}$

$h=-6 \rightarrow 6$

$k=-10 \rightarrow 10$

$l=-17 \rightarrow 17$

Secondary atom site location: difference Fourier map

Hydrogen site location: inferred from neighbouring sites

$\mathrm{H}$-atom parameters constrained

$w=1 /\left[\sigma^{2}\left(F_{\mathrm{o}}^{2}\right)+(0.0872 P)^{2}+0.2177 P\right]$

where $P=\left(F_{\mathrm{o}}^{2}+2 F_{\mathrm{c}}^{2}\right) / 3$

$(\Delta / \sigma)_{\max }<0.001$

$\Delta \rho_{\max }=1.15 \mathrm{e} \AA^{-3}$

$\Delta \rho_{\min }=-0.38$ e $\AA^{-3}$

\section{Special details}

Geometry. All e.s.d.'s (except the e.s.d. in the dihedral angle between two 1.s. planes) are estimated using the full covariance matrix. The cell e.s.d.'s are taken into account individually in the estimation of e.s.d.'s in distances, angles and torsion angles; correlations between e.s.d.'s in cell parameters are only used when they are defined by crystal symmetry. An approximate (isotropic) treatment of cell e.s.d.'s is used for estimating e.s.d.'s involving l.s. planes.

Refinement. Refinement of $F^{2}$ against ALL reflections. The weighted $R$-factor $w R$ and goodness of fit $S$ are based on $F^{2}$, conventional $R$-factors $R$ are based on $F$, with $F$ set to zero for negative $F^{2}$. The threshold expression of $F^{2}>\sigma\left(F^{2}\right)$ is used only for calculating $R$-factors $(\mathrm{gt})$ etc. and is not relevant to the choice of reflections for refinement. $R$-factors based on $F^{2}$ are statistically about twice as large as those based on $F$, and $R$-factors based on ALL data will be even larger.

Fractional atomic coordinates and isotropic or equivalent isotropic displacement parameters $\left(\AA^{2}\right)$

\begin{tabular}{lllll}
\hline & $x$ & $y$ & $z$ & $U_{\text {iso }} * / U_{\text {eq }}$ \\
\hline Br1 & $0.70371(17)$ & $0.78972(8)$ & $0.05167(5)$ & $0.0774(4)$ \\
$\mathrm{S} 1$ & $0.3354(2)$ & $0.11701(17)$ & $0.32101(10)$ & $0.0365(4)$ \\
O1 & $0.2650(7)$ & $0.0106(5)$ & $0.2528(3)$ & $0.0486(10)$ \\
O2 & $0.1387(7)$ & $0.1738(5)$ & $0.3930(3)$ & $0.0492(10)$ \\
O3 & $0.7775(7)$ & $-0.4424(5)$ & $0.4169(3)$ & $0.0505(10)$ \\
$\mathrm{H} 3 \mathrm{O}$ & 0.7174 & -0.5221 & 0.4536 & $0.076^{*}$ \\
$\mathrm{O} 4$ & $0.4156(7)$ & $-0.2871(4)$ & $0.4743(3)$ & $0.0427(9)$ \\
$\mathrm{N} 1$ & $0.5998(8)$ & $0.0162(5)$ & $0.3816(3)$ & $0.0389(10)$ \\
$\mathrm{H} 1$ & 0.6632 & 0.0630 & 0.4278 & $0.047 *$ \\
$\mathrm{C} 1$ & $0.5927(12)$ & $0.5917(7)$ & $0.1309(4)$ & $0.0456(14)$ \\
$\mathrm{C} 2$ & $0.3808(12)$ & $0.6089(7)$ & $0.1979(5)$ & $0.0495(15)$ \\
$\mathrm{H} 2$ & 0.2914 & 0.7172 & 0.2039 & $0.059 *$ \\
$\mathrm{C} 3$ & $0.3022(11)$ & $0.4635(7)$ & $0.2560(5)$ & $0.0453(14)$ \\
$\mathrm{H} 3$ & 0.1575 & 0.4737 & 0.3010 & $0.054 *$
\end{tabular}




$\begin{array}{lllll}\text { C4 } & 0.4368(10) & 0.3039(7) & 0.2478(4) & 0.0355(12) \\ \text { C5 } & 0.6490(11) & 0.2867(7) & 0.1801(4) & 0.0489(15) \\ \text { H5 } & 0.7386 & 0.1784 & 0.1742 & 0.059^{*} \\ \text { C6 } & 0.7271(13) & 0.4320(8) & 0.1211(5) & 0.0584(17) \\ \text { H6 } & 0.8695 & 0.4221 & 0.0751 & 0.070^{*} \\ \text { C7 } & 0.7389(10) & -0.1470(6) & 0.3652(4) & 0.0410(13) \\ \text { H7A } & 0.9274 & -0.1559 & 0.3839 & 0.049^{*} \\ \text { H7B } & 0.7316 & -0.1519 & 0.2925 & 0.049^{*} \\ \text { C8 } & 0.6253(10) & -0.2978(7) & 0.4249(4) & 0.0368(12)\end{array}$

Atomic displacement parameters $\left(\AA^{2}\right)$

\begin{tabular}{lllllll}
\hline & $U^{11}$ & $U^{22}$ & $U^{33}$ & $U^{12}$ & $U^{13}$ & $U^{23}$ \\
\hline Br1 & $0.1144(7)$ & $0.0372(4)$ & $0.0728(6)$ & $-0.0176(4)$ & $0.0098(4)$ & $0.0154(3)$ \\
S1 & $0.0376(7)$ & $0.0264(7)$ & $0.0458(8)$ & $-0.0085(5)$ & $0.0029(5)$ & $-0.0043(6)$ \\
O1 & $0.053(2)$ & $0.037(2)$ & $0.060(3)$ & $-0.0124(18)$ & $-0.0073(18)$ & $-0.0143(19)$ \\
O2 & $0.045(2)$ & $0.046(2)$ & $0.058(2)$ & $-0.0118(18)$ & $0.0163(18)$ & $-0.011(2)$ \\
O3 & $0.050(2)$ & $0.027(2)$ & $0.069(3)$ & $0.0001(18)$ & $0.0167(19)$ & $-0.001(2)$ \\
O4 & $0.0398(19)$ & $0.027(2)$ & $0.059(2)$ & $-0.0058(16)$ & $0.0127(17)$ & $-0.0008(17)$ \\
N1 & $0.050(2)$ & $0.019(2)$ & $0.046(3)$ & $-0.0062(19)$ & $-0.002(2)$ & $-0.001(2)$ \\
C1 & $0.064(4)$ & $0.026(3)$ & $0.044(3)$ & $-0.009(3)$ & $-0.004(3)$ & $0.002(3)$ \\
C2 & $0.059(3)$ & $0.017(3)$ & $0.068(4)$ & $0.003(3)$ & $0.000(3)$ & $-0.006(3)$ \\
C3 & $0.045(3)$ & $0.025(3)$ & $0.065(4)$ & $-0.002(2)$ & $0.008(3)$ & $-0.007(3)$ \\
C4 & $0.041(3)$ & $0.027(3)$ & $0.038(3)$ & $-0.003(2)$ & $-0.001(2)$ & $-0.005(2)$ \\
C5 & $0.064(4)$ & $0.022(3)$ & $0.054(4)$ & $0.001(3)$ & $0.013(3)$ & $0.004(3)$ \\
C6 & $0.073(4)$ & $0.040(4)$ & $0.054(4)$ & $-0.001(3)$ & $0.021(3)$ & $0.006(3)$ \\
C7 & $0.039(3)$ & $0.032(3)$ & $0.049(3)$ & $-0.010(2)$ & $0.009(2)$ & $0.001(3)$ \\
C8 & $0.035(3)$ & $0.033(3)$ & $0.042(3)$ & $-0.007(2)$ & $0.000(2)$ & $-0.003(2)$ \\
& & & & & & \\
\hline
\end{tabular}

Geometric parameters $\left(\stackrel{A}{\circ}{ }^{o}\right)$

\begin{tabular}{llll}
\hline $\mathrm{Br} 1-\mathrm{C} 1$ & $1.886(5)$ & $\mathrm{C} 2-\mathrm{C} 3$ & $1.379(7)$ \\
$\mathrm{S} 1-\mathrm{O} 1$ & $1.429(4)$ & $\mathrm{C} 2-\mathrm{H} 2$ & 0.9300 \\
$\mathrm{~S} 1-\mathrm{O} 2$ & $1.436(4)$ & $\mathrm{C} 3-\mathrm{C} 4$ & $1.374(7)$ \\
$\mathrm{S} 1-\mathrm{N} 1$ & $1.594(4)$ & $\mathrm{C} 3-\mathrm{H} 3$ & 0.9300 \\
$\mathrm{~S} 1-\mathrm{C} 4$ & $1.765(5)$ & $\mathrm{C} 4-\mathrm{C} 5$ & $1.380(7)$ \\
$\mathrm{O} 3-\mathrm{C} 8$ & $1.306(6)$ & $\mathrm{C} 5-\mathrm{C} 6$ & $1.382(7)$ \\
$\mathrm{O} 3-\mathrm{H} 3 \mathrm{C}$ & 0.8200 & $\mathrm{C} 5-\mathrm{H} 5$ & 0.9300 \\
$\mathrm{O} 4-\mathrm{C} 8$ & $1.223(6)$ & $\mathrm{C} 6-\mathrm{H} 6$ & 0.9300 \\
$\mathrm{~N} 1-\mathrm{C} 7$ & $1.436(6)$ & $\mathrm{C} 7-\mathrm{C} 8$ & $1.499(6)$ \\
$\mathrm{N} 1-\mathrm{H} 1$ & 0.8600 & $\mathrm{C} 7-\mathrm{H} 7 \mathrm{~A}$ & 0.9700 \\
$\mathrm{C} 1-\mathrm{C} 2$ & $1.373(8)$ & $\mathrm{C} 7-\mathrm{H} 7 \mathrm{~B}$ & 0.9700 \\
$\mathrm{C} 1-\mathrm{C} 6$ & $1.379(8)$ & & $120.5(5)$ \\
& & & $120.6(4)$ \\
$\mathrm{O} 1-\mathrm{S} 1-\mathrm{O} 2$ & $119.3(2)$ & $\mathrm{C} 3-\mathrm{C} 4-\mathrm{C} 5$ & $118.9(4)$ \\
$\mathrm{O} 1-\mathrm{S} 1-\mathrm{N} 1$ & $106.7(2)$ & $\mathrm{C} 3-\mathrm{C} 4-\mathrm{S} 1$ & $119.4(5)$ \\
$\mathrm{O} 2-\mathrm{S} 1-\mathrm{N} 1$ & $109.3(2)$ & $\mathrm{C} 5-\mathrm{C} 4-\mathrm{S} 1$ & \\
$\mathrm{O} 1-\mathrm{S} 1-\mathrm{C} 4$ & $108.9(2)$ & $\mathrm{C} 4-\mathrm{C} 5-\mathrm{C} 6$ &
\end{tabular}




\begin{tabular}{|c|c|c|c|}
\hline $\mathrm{O} 2-\mathrm{S} 1-\mathrm{C} 4$ & $106.5(2)$ & $\mathrm{C} 4-\mathrm{C} 5-\mathrm{H} 5$ & 120.3 \\
\hline $\mathrm{N} 1-\mathrm{S} 1-\mathrm{C} 4$ & $105.3(2)$ & $\mathrm{C} 6-\mathrm{C} 5-\mathrm{H} 5$ & 120.3 \\
\hline $\mathrm{C} 8-\mathrm{O} 3-\mathrm{H} 3 \mathrm{O}$ & 109.5 & $\mathrm{C} 1-\mathrm{C} 6-\mathrm{C} 5$ & $119.6(5)$ \\
\hline $\mathrm{C} 7-\mathrm{N} 1-\mathrm{S} 1$ & $124.8(4)$ & $\mathrm{C} 1-\mathrm{C} 6-\mathrm{H} 6$ & 120.2 \\
\hline $\mathrm{C} 7-\mathrm{N} 1-\mathrm{H} 1$ & 117.6 & $\mathrm{C} 5-\mathrm{C} 6-\mathrm{H} 6$ & 120.2 \\
\hline $\mathrm{S} 1-\mathrm{N} 1-\mathrm{H} 1$ & 117.5 & $\mathrm{~N} 1-\mathrm{C} 7-\mathrm{C} 8$ & $113.8(4)$ \\
\hline $\mathrm{C} 2-\mathrm{C} 1-\mathrm{C} 6$ & $121.0(5)$ & $\mathrm{N} 1-\mathrm{C} 7-\mathrm{H} 7 \mathrm{~A}$ & 108.8 \\
\hline $\mathrm{C} 2-\mathrm{C} 1-\mathrm{Br} 1$ & $119.6(4)$ & $\mathrm{C} 8-\mathrm{C} 7-\mathrm{H} 7 \mathrm{~A}$ & 108.8 \\
\hline $\mathrm{C} 6-\mathrm{C} 1-\mathrm{Br} 1$ & $119.4(4)$ & $\mathrm{N} 1-\mathrm{C} 7-\mathrm{H} 7 \mathrm{~B}$ & 108.8 \\
\hline $\mathrm{C} 1-\mathrm{C} 2-\mathrm{C} 3$ & $119.2(5)$ & $\mathrm{C} 8-\mathrm{C} 7-\mathrm{H} 7 \mathrm{~B}$ & 108.8 \\
\hline $\mathrm{C} 1-\mathrm{C} 2-\mathrm{H} 2$ & 120.4 & $\mathrm{H} 7 \mathrm{~A}-\mathrm{C} 7-\mathrm{H} 7 \mathrm{~B}$ & 107.7 \\
\hline $\mathrm{C} 3-\mathrm{C} 2-\mathrm{H} 2$ & 120.4 & $\mathrm{O} 4-\mathrm{C} 8-\mathrm{O} 3$ & $124.3(5)$ \\
\hline $\mathrm{C} 4-\mathrm{C} 3-\mathrm{C} 2$ & $120.2(5)$ & $\mathrm{O} 4-\mathrm{C} 8-\mathrm{C} 7$ & $124.4(5)$ \\
\hline $\mathrm{C} 4-\mathrm{C} 3-\mathrm{H} 3$ & 119.9 & $\mathrm{O} 3-\mathrm{C} 8-\mathrm{C} 7$ & $111.4(4)$ \\
\hline $\mathrm{C} 2-\mathrm{C} 3-\mathrm{H} 3$ & 119.9 & & \\
\hline $\mathrm{O} 1-\mathrm{S} 1-\mathrm{N} 1-\mathrm{C} 7$ & $2.2(4)$ & $\mathrm{O} 1-\mathrm{S} 1-\mathrm{C} 4-\mathrm{C} 5$ & $-57.6(5)$ \\
\hline $\mathrm{O} 2-\mathrm{S} 1-\mathrm{N} 1-\mathrm{C} 7$ & $132.5(4)$ & $\mathrm{O} 2-\mathrm{S} 1-\mathrm{C} 4-\mathrm{C} 5$ & $172.6(4)$ \\
\hline $\mathrm{C} 4-\mathrm{S} 1-\mathrm{N} 1-\mathrm{C} 7$ & $-113.5(4)$ & $\mathrm{N} 1-\mathrm{S} 1-\mathrm{C} 4-\mathrm{C} 5$ & $56.6(5)$ \\
\hline $\mathrm{C} 6-\mathrm{C} 1-\mathrm{C} 2-\mathrm{C} 3$ & $0.0(9)$ & $\mathrm{C} 3-\mathrm{C} 4-\mathrm{C} 5-\mathrm{C} 6$ & $0.6(9)$ \\
\hline $\mathrm{Br} 1-\mathrm{C} 1-\mathrm{C} 2-\mathrm{C} 3$ & $-179.5(4)$ & $\mathrm{S} 1-\mathrm{C} 4-\mathrm{C} 5-\mathrm{C} 6$ & $178.9(5)$ \\
\hline $\mathrm{C} 1-\mathrm{C} 2-\mathrm{C} 3-\mathrm{C} 4$ & $0.8(9)$ & $\mathrm{C} 2-\mathrm{C} 1-\mathrm{C} 6-\mathrm{C} 5$ & $-0.5(10)$ \\
\hline $\mathrm{C} 2-\mathrm{C} 3-\mathrm{C} 4-\mathrm{C} 5$ & $-1.1(8)$ & $\mathrm{Br} 1-\mathrm{C} 1-\mathrm{C} 6-\mathrm{C} 5$ & $179.1(5)$ \\
\hline $\mathrm{C} 2-\mathrm{C} 3-\mathrm{C} 4-\mathrm{S} 1$ & $-179.4(4)$ & $\mathrm{C} 4-\mathrm{C} 5-\mathrm{C} 6-\mathrm{C} 1$ & $0.2(9)$ \\
\hline $\mathrm{O} 1-\mathrm{S} 1-\mathrm{C} 4-\mathrm{C} 3$ & $120.7(5)$ & $\mathrm{S} 1-\mathrm{N} 1-\mathrm{C} 7-\mathrm{C} 8$ & $-85.5(5)$ \\
\hline $\mathrm{O} 2-\mathrm{S} 1-\mathrm{C} 4-\mathrm{C} 3$ & $-9.1(5)$ & $\mathrm{N} 1-\mathrm{C} 7-\mathrm{C} 8-\mathrm{O} 4$ & $8.2(8)$ \\
\hline $\mathrm{N} 1-\mathrm{S} 1-\mathrm{C} 4-\mathrm{C} 3$ & $-125.2(5)$ & $\mathrm{N} 1-\mathrm{C} 7-\mathrm{C} 8-\mathrm{O} 3$ & $-172.4(4)$ \\
\hline
\end{tabular}

Hydrogen-bond geometry $\left(\AA,{ }^{\circ}\right)$

\begin{tabular}{lllll}
\hline$D-\mathrm{H} \cdots A$ & $D-\mathrm{H}$ & $\mathrm{H} \cdots A$ & $D \cdots A$ & $D-\mathrm{H} \cdots A$ \\
\hline $\mathrm{O} 3-\mathrm{H} 3 O \cdots \mathrm{O} 4^{\mathrm{i}}$ & 0.82 & 1.85 & $2.671(5)$ & 174 \\
$\mathrm{~N} 1-\mathrm{H} 1 \cdots \mathrm{O} 4^{\mathrm{ii}}$ & 0.86 & 2.38 & $3.124(5)$ & 146 \\
$\mathrm{C} 2-\mathrm{H} 2 \cdots 1^{\mathrm{iii}}$ & 0.93 & 2.53 & $3.384(7)$ & 153 \\
$\mathrm{C} 3-\mathrm{H} 3 \cdots \mathrm{O} 3^{\text {iv }}$ & 0.93 & 2.50 & $3.423(7)$ & 170 \\
$\mathrm{C} 3-\mathrm{H} 3 \cdots \mathrm{O} 2$ & 0.93 & 2.50 & $2.884(7)$ & 105 \\
\hline
\end{tabular}

Symmetry codes: (i) $-x+1,-y-1,-z+1$; (ii) $-x+1,-y,-z+1$; (iii) $x, y+1, z$; (iv) $x-1, y+1, z$. 